\title{
A BIBLIOMETRIC CONTENT ANALYSIS FOR UNDERSTANDING 5G TECHNOLOGY: PROMISE, CHALLENGES AND FUTURE ROAD MAP
}

\author{
${ }^{* 1}$ Garima Verma, ${ }^{2}$ Hemraj Verma \\ ${ }^{*}$ School of Computing, DIT University, Dehradun, INDIA \\ Mobile: 9410148069, *Email:garimaverma.research@gmail.com \\ ${ }^{2}$ Faculty of Management Studies, DIT University, Dehradun, INDIA
}

Email: hemraj77@gmail.com

\begin{abstract}
Purpose - The main purpose of this paper is to bring a comprehensive understanding of 5G mobile communication technology, its comparison with predecessor technologies, and research issues related to its implementation. Further, this study also attempts to find out its key drivers, potential roadblocks, and benefits related to this technology. It will also suggest a future research road map in this area.

Design/methodology/approach - An in-depth literature review and content analysis of key reports and articles published since 2014 have been carried out to achieve stated research objectives.

Findings - The findings suggest that 5G technology holds a lot of promise but there are challenges of security $\&$ privacy, spectrum availability, switching cost, slow infrastructure development, etc., which need to be taken care of before 5G is successfully adopted.

Originality/value - The current research uses an in-depth literature review of all key reports and journal articles that have been published since 2014. Further, it highlights the major issues and challenges that exist for 5G implementation.
\end{abstract}

Keywords: 5G Technology, In-depth Literature review, content analysis, security, privacy.

\section{Introduction}

Cellular mobile communication has been changing rapidly with newer generation of technologies redefining how users would experience communication. From 2G(GMS) to 3G(UMTS), 3G to 4G (LTE-A/ WiMAX), and now from 4G to $5 \mathrm{G}$, the world appears to be getting ready to embrace fifth generation cellular mobile communication [1], [2]. Mobile communication technologies, identified as a generation, have evolved from $1 \mathrm{G}$ to $5 \mathrm{G}$ today, with each generation of technology immensely scaling up the data processing speed and functionalities in a smartphone [3], [4]. 1G i.e. first generation only provided functionality of analogue voice calls which got extended in $2 \mathrm{G}$ with an added option of transmitting text messages on basis of digital data transfer [5].

$3 \mathrm{G}$ communication technology offered a lot more in the form of faster data exchange rate, availability of multimedia services and better network coverage [6]. After 3G, the world moved to 4G communication technology, also known as LTE and LTE-A, which provided far more better functionalities at a very good data rate and internet speed. User can now watch live videos, use video calls, and do much more at a fairly good speed. There has been a constantly increasing demand for ubiquitous connectivity and high data exchange rates for last two decades causing scarcity of spectrum [7]. With everincreasing demand for seamless data, 5G holds a lot of promises for significantly improved user experience through a very high speed of voluminous data exchange, reduced latency and extremely fast internet. It is believed to increase efficiencies in energy, spectrum, network etc. [5]. It is expected to bring in huge revenue as $5 \mathrm{G}$ will open doors for a lot of newer applications and functionalities. As per Ericsson Mobility Report [3], the total number of mobile 5G subscribers is expected to reach 1.9 billion, 45 percent of world population will be using $5 \mathrm{G}$ and $5 \mathrm{G}$ networks shall carry 35 percent of world traffic by 2024 . Even region-wise projections indicate a positive outlook. North America has already commercialized $5 \mathrm{G}$ mobile and it is expected that over 270 million users will join fixed wireless services by this time period. Western Europe, Central Europe and Eastern Europe, had around $40 \%$ mobile subscribers after 5G launch in 2019. North-East Asia and India, too, are expected to have 5G subscriber base of $47 \%$ and $6 \%$ respectively by 2024 . 
Also, technologies such as NB-IoT and Cat-M are expected to have $45 \%$ of IoT connections by the same time period. With all these predictions, 5G looks like an inevitability. However, this euphoria and excitement around 5G launch, growth and penetration may be premature as majority of markets around the world, except North America, West Europe and East Asia, are not ready fully for enjoying $5 \mathrm{G}$ mobile functionalities. For example, in India, most of the rural population is yet to exploit $4 \mathrm{G}$ cellular functionalities fully, largely due to lack of infrastructure and spectrum issues. The similar situation is found in most of the developing and under-developed countries as well. Therefore, it would not be a surprise if $5 \mathrm{G}$ mobile technology takes longer than the usual time [3], [5].

Despite all the roadblocks and issues, communication technologies remain an important areas of research. A lot of research studies have been done on the mobile communication technologies facilitating its evolution from $1 \mathrm{G}$ to $5 \mathrm{G}$. Since, $5 \mathrm{G}$ is the latest generation of communication technologies and it is yet to be fully launched, therefore, researchers around the world have started their research work on $5 \mathrm{G}$ enabled technologies [8]. 5G will use millimeter wave (mmWave) technology and many other technologies such as multiple-input multiple-output (MIMO), cloud-based network, Cognitive Radio(CR) technology, device to device (D2D) communications etc. [10]. Cognitive radio (CR) technology is one such technology that is used in $5 \mathrm{G}$ to provide a possibility for increasing the spectrum efficiency of 5G. Cognitive radio based $5 \mathrm{G}$ provides a very high data rates and Quality of Service (QoS) applications [9]. Similarly, the hybrid mobile femtocells [11] which is proposed to be based on mmWaves technology, is likely to provide the solution for $5 \mathrm{G}$ vehicles.

Further, security is also one of the requirement for successful $5 \mathrm{G}$ implementation. There are various security technologies such as block chain, network slicing, encryption, etc. [13], [14] which have been proposed for making 5G more secure and robust.

Apart from all above technologies, there are other technologies such as software defined networking (SDN) and Network function virtualization (NFV) which are used for intelligent manufacturing concept [15], [16]. SDN can be used to reduce the operating cost and load of equipment and NFV uses the concept of centralization of hardware such as storage, servers, etc. [17].

The expectations from $5 \mathrm{G}$ networks are quite high as it is likely to provide high data rates, flexible platform (for full IoT, Natural Language Processing (NLP) and cloud computing), ultra-reliability, low latency $(<1 \mathrm{~ms})$, extended battery life etc. [12]. Further, it is expected to provide reasonable high throughput than long term evolution (LTE) in milliseconds. Also, 5G networks technology offer manufacturers and industrialist the opportunity to build smart factories, which will use advantages of various technologies such as automation, AI, troubleshooting by augmented reality, and IoT, etc. [14].

Since $5 \mathrm{G}$ communication is still in testing phases around the world, therefore, 5G and its enabling communication technologies discussed above are likely to offer a wide scope for carrying our research on variety of issues. It would interesting, timely and highly relevant to examine $5 \mathrm{G}$ promise, challenges and future prospects by undertaking indepth literature review and analysis.

In view of the above, this research study makes an attempt to understand 5G communication technology, the possible implementation challenges and future prospects it holds for end users if implemented successfully. Specifically, the study aims to understand and discuss -

1. What $5 \mathrm{G}$ technology is and how is it different from its predecessors.

2. Key enabling Technologies of 5G.

3. Architectural requirements of $5 \mathrm{G}$ technology.

4. Key research areas related to 5G technology research.

5. Major challenges for implementation of 5G technology.

The remaining paper has been organized into four sections. Section 2 presents the methodology used for review and analysis. Section 3 describes the findings and discussions based on the analysis done in the previous sections, and in the last section 4 conclusion of the work is covered.

\section{Methodology}

This research study attempts to answer research questions by relying on exploratory research design. In this, primarily two exploratory techniques viz. systematic literature review and content analysis have been used. Most of the research questions have been addressed using systematic review technique whereas for one question, bibliometric content analysis was deemed more appropriate [18]. Using first technique, the extant literature available related to $5 \mathrm{G}$ technology has been reviewed in detail. Literature review is considered as one of the higher level of research evidence in other empirical testimonies as there is an element of analysis 
and synthesis in it [19]. Systematic reviews, which are different from ad-hoc reviews, has been recognized as a scientific activity and are being applied in other areas such as production economics too [20], [21], [22]. Further, a threestage protocol of gathering relevant literature, analysis and synthesizing data and drawing meaningful implications, as suggest by Transfield et al., [18] was used for the study. Further, critical content analysis technique has been used to find out key research focal areas in 5G technology.

\subsection{Collection of literature data}

In this phase, in-depth literature review of all articles has been done which are published in journals, conference proceedings and reports since 2010. Most of the articles have been taken from database such as sciendirect.com, Springer, and IEEE. A critical content analysis was conducted for all the reports and research papers. The Fig. 1 shows the systematic flowchart of collection of literature data. Since quantitative and qualitative approach, alone, is not sufficient to analyze all aspects of the contents [23], [24], [25], therefore, the technique of Critical Content Analysis (CCA), an approach that involves quantitative as well as qualitative analysis, was undertaken to provide deeper analysis and obtain more appropriate findings [26].

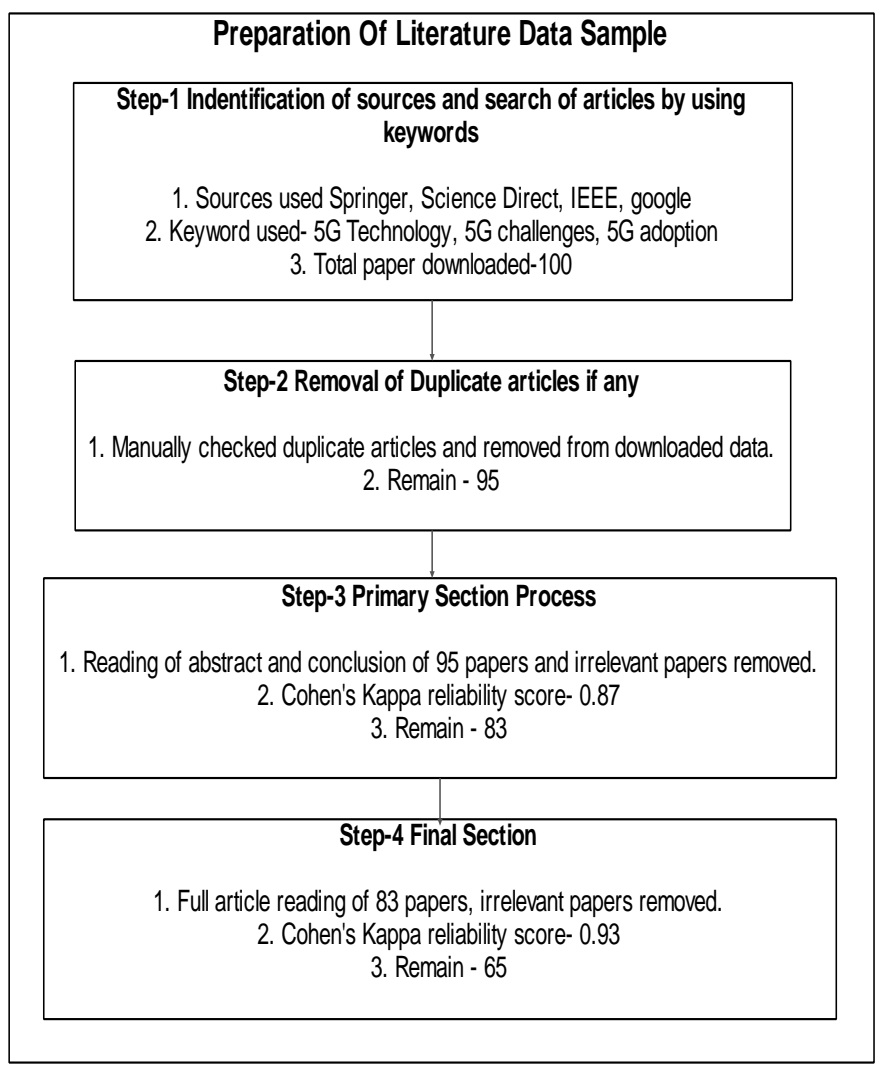

Fig. 1 Flowchart for collection of literature data

\section{Findings and Discussions}

This study performed systematic view of literature to meet research objectives $1,2,3$, and 4 . The details of the same are as below:

\section{Objective 1: To understand what 5G Technology is and how it is different from its predecessors.}

The fifth generation (5G) technology is expected to be a significant improvement over its predecessor technologies in terms of wider bandwidth per frequency channel over a new frequency band [8]. This advanced technology can be better understood in terms of evolution that has happened over the period of time in communication technology. This evolution of communication technology from $1 \mathrm{G}$ through $5 \mathrm{G}$ has happened primarily because of the requirement gap which a predecessor technology could not meet [5]. For example, when users communicated through $1 \mathrm{G}$ technology, they could only talk using analogues technology. But this technology suffered from poor spectral efficiency and has major security issues with it. So when a need for a more secure and better spectral efficiency was felt, $2 \mathrm{G}$ technology was developed in which users could make a call using digital signals and also send messages. However, this second generation technology could cover some limitations (security) of $1 \mathrm{G}$ but lacked in terms of limited data rates thus making use of internet and sending emails difficult [2]. After this, $3^{\text {rd }}$ Generation communication technology was launched and made internet usage a far better experience. In addition to makings phone calls and messaging, a user could surf the net at a faster speed, watch videos, listen songs and do lot more for entertainment and other purposes too. However, the initial hype of fast internet failed as users could not access internet over WAP. This led to launch of $3.5 \mathrm{G}$ which was a technology based on broad band. It indeed overcame most of the problems of all predecessors but suffered from rigid architecture and protocols [2], [3]. This led to more advancements and 4G was born, with lots of expectations. User now could do so many things, not only on computers but also on their smartphones. This led to usage of apps and social media. Entertainment took the center stage along with several new applications that were relevant to businesses also. So $4 \mathrm{G}$ brought a faster broadband based internet where users could live stream videos, share heavy files, images, videos etc. at ease bringing huge benefits to almost all stakeholders i.e. end users, operators and third party owners as well [27]. But as the 
cheap data usage requirements increased exponentially, even $4 \mathrm{G}$ appeared to have reached its limits. This led mobile communication firms to look for even better alternative which gave birth to fifth generation (5G) Technology.

Initially, there were two views that emerged for understanding what $5 \mathrm{G}$ technology is - the first view was hyper-connected vision in which $5 \mathrm{G}$ technology would be created out of a blend of its predecessor technologies such as $1 \mathrm{G}, 2 \mathrm{G}, 3 \mathrm{G}$ and $4 \mathrm{G}$ for better coverage and speed through more network density thus allowing communication between machine-to-machine and Internet of things. Further, this view was based on radio access technology resulting in lower power and low throughput devices.

The second view on 5G technology was based on advanced radio access technology with targeted data rates and latency as a means to demarcate other predecessors from 5G [28]. Despite having two different views, these are, today, seen as a single set for the progression of mobile communication technology. In short, $5 \mathrm{G}$ technology will have an increased bit rate supporting upto $10 \mathrm{Gbps}$ speed, very high peak bit rate, concurrent connectivity of more devices, more volume of data per unit area, more battery efficiency, better and consistent geographic coverage, lower cost for developing infrastructure[29]. Some of the key features of 5G technology are summarized as below -

- Ultra high speed of 1 to $10 \mathrm{Gbps}$

- Latency close to zero (1 millisecond with real time video streaming).

- $1,000 \mathrm{x}$ bandwidth for faster response and high capacity.

- Concurrent connectivity of 10 to 100 devices.

- Worldwide coverage and seamless connectivity.

- Highly energy efficiency - close to $90 \%$ reduction of energy usage in the network.

- Longer Battery life

- Global wi-fi zones.

- Wide range of applications

For users, there are several benefits such possibility of using multiple services (listen watch movie and play games while talking over phone) and control PC with smartphone. A doctor may treat patients in any part of the globe easily, a student may learn from any part of the world, govt. may monitor anyone anywhere, locate missing objects, predict for natural disasters. To put simply, $5 \mathrm{G}$ technology is a next generation technology that will potentially revolutionize communication. It will change the way how human beings will communicate and entertain. It promises to have huge number of innovative features making your smartphone your laptop [30]. With zero latency and seamless connectivity, users would experience a new world where they can simultaneously do several things such as playing games, receiving calls, watching videos or use other multimedia options without any problem

\section{Objective 2: To Understand Key enabling technologies of 5G.}

To deploy 5G technology, there are different enabling technologies such as Radio Access Networks (RAN), core networks such as network functional virtualization (NFV), software defined networking (SDN), network slicing and Cloud-RAN (C-RAN), Black haul networks and Front Haul links. These are discussed as below -

i. Radio Access Networks (RAN) - The radio access networks developed for $4 \mathrm{G}$, will have densification by deploying small cells covering much smaller area as compared to a macro cell leading to wider network coverage, increased capacity (without any need for additional spectrum) and improved quality of service. This will make 5G and enhanced broad band services possible. The small cells will carry small cell antennae at bus shelter, traffics lights etc. making increased data rate possible [11].

ii. Core Networks - For 5G, core networks such as network functional virtualization (NFV), software defined networking (SDN), network slicing and Cloud-RAN (C-RAN) are required. C-RAN, which is a cloud based radio access network, uses virtualization technique to make it possible to keeping pace with innovation and mobile network transformation. NFV replaces functions of the network on dedicate devices such routers, firewalls and load balancers. SDN makes dynamic reconfiguration of elements in the elements possible in real time leading to improved performance and service quality [14].

iii. Backhaul - Backhaul networks are required to achieve super speed and high capacity as needed in 5G. It connects the radio access network (RAN) to the core network.

iv. Front haul- As per the definition of recommendation ITU-T Y.3100 Front haul is a network path between Remote radio units and centralized radio controllers 
of a function of base station. It helps in reducing latency and bandwidth requirements [2].

\section{Objective 3: To Understand Architectural requirements of $5 \mathrm{G}$ technology.}

In the present LTE which is called as $4 \mathrm{G}$ system, the scalability in network traffic eruption is limited. The reason for this is the main network of LTE has centralized and hierarchical architecture in which the Packet Data Network (PDN) gateway is fixed between the mobile terminal and external packet networks. A simple architecture of $5 \mathrm{G}$ is shown in Fig. 2. While in upcoming $5 \mathrm{G}$ system, to support thousand times of traffic distributed architecture will be used for implementation. Apart from this $5 \mathrm{G}$ will support multiRAT technologies which will provide high volume data in low cost with improved accuracy. 5G architecture will also have trace the device location functionality [1], [2].

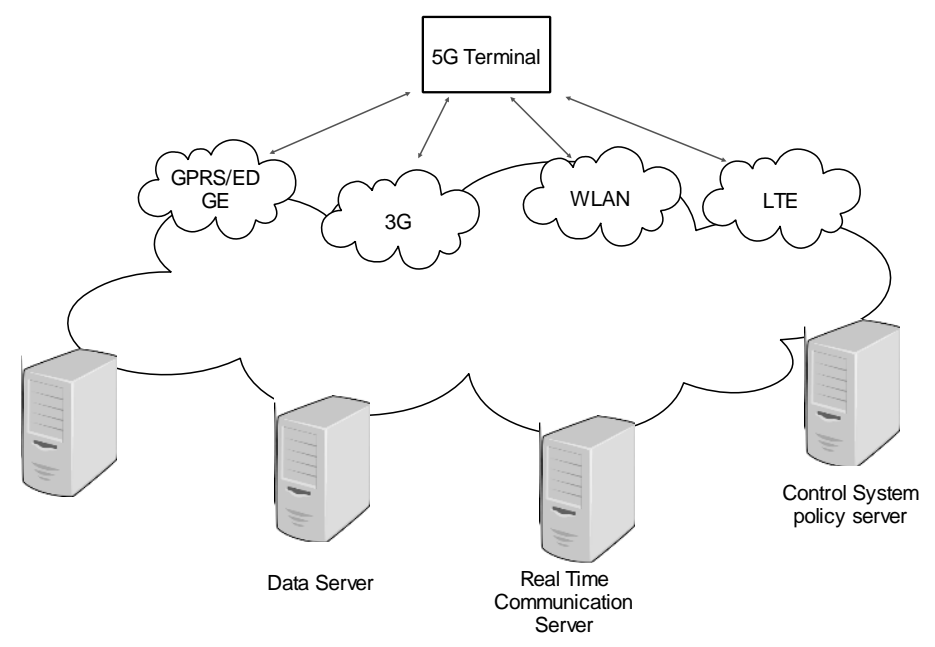

Fig. 2 Simple architecture of 5G

The 5G model is based on Internet Protocol (IP). The main part of $5 \mathrm{G}$ architecture is user terminal which consists with autonomous independent radio technologies. These radio technologies act as an IP link between outside internet worlds [29]. The IP technology ensures the sessions between client and server applications on the internet. Some major wireless network requirements in $5 \mathrm{G}$ are spectral efficiency, which is 3 times higher than $4 \mathrm{G}$, peak data rate, approximately $20 \mathrm{Gbps}$ for $5 \mathrm{G}$, user experience data rate is $100 \mathrm{Mbps} / 1 \mathrm{Gbps}$, etc. [29], [30].

\section{Objective 4: To Understand Key areas of research related to $5 \mathrm{G}$ technology.}

To achieve the stated objective, critical content analysis approach was used. To perform content analysis, four categories - Reports, white papers, journal articles and conference papers were included in the study from various digital sources such as Springer digital library, Sciencedirect.com, IEEExplore, and Google. In the initial stage total 100 papers were downloaded by searching of keywords 5G technology, 5G challenges etc. After downloading, the articles were checked for duplicate articles and found 5 articles downloaded two times. After removal of 5 articles, total papers included for the analysis were 95 . With remaining 95 articles authors started reading abstract and conclusion of each articles to find relevant articles among 95. Out of these 95 articles, 12 were found irrelevant and ,therefore, were removed from the study sample [31]. The Cohen's kappa reliability score was calculated as 0.87 . Finally, 83 articles were included for next stage processing. After selecting 83 articles, final selection process was done by reading all the papers full to find the most relevant papers for the study. In this stage, a total 18 papers were found as irrelevant and ,therefore, were removed from the sample. The value of Cohen's kappa reliability score was calculated as 0.93 and final left sample was 65 papers.

\section{Data Analysis and Findings-}

After collection of data from literature i.e. 65 articles on 5G technology. The bibliographic analysis method was used. This method uses co-occurrence networks which, represents quantitative content based literature data [22]. In recent state of arts this method is identified as an effective method to represent literature data graphically by dividing it into clusters, to explore the recent trends. The graphical representation of data uses count of words used in the articles. The co-occurrence network of keywords has been implemented using VOSViewer software [32], [33]. This software does mining the dataset of keywords and their occurrences in the numbers of papers according to defined threshold value set by the user. For the study the threshold value was set as 2 for keywords analysis. All the keywords in the dataset that have satisfied the threshold value were mapped according to clusters.

For research objective 4, all the keywords found from the articles and after reviewing all the reports and research papers on 5G mobile technologies- 
Out of 118 only 41 keywords met the threshold. But there were some keywords found duplicate in the form of writing style example QoS or Quality of Service etc., some were found irrelevant etc. finally only 26 relevant keywords has been selected. For remaining 26 keywords using full counting and normalization of association algorithm, VOSViewer software has calculated the strength of links between cooccurrence networks. The keywords which had higher rank impacted the formation of clusters [31]. Total 5 clusters has been formed and named on the basis of two highest count of keyword in the cluster- (a) Machine to Machine (M2M) and QoS, (b) MIMO and SDN, (c) IoT and Security, (d) Low Latency and mmWaves, (e) Virtual Reality and Cognitive Radio. The VOSViewer output is shown in Fig. 3. The circles are representing the keywords and the color represents the groups of related items. Keywords are located on the basis of co-occurrence in the papers, higher the number of cooccurrences the closely they are located in the map. The terms exists in the cluster (a) occurred in 33 articles out of 65 selected one, in cluster (b) the terms occurred in 34 articles, in cluster (c) the terms occurred in 42 articles, in cluster (d) the terms occurred in 19 articles and in cluster (e) the terms occurred in all 65 articles. Among all terms the low latency and IoT has occurred in almost all the articles. Table- 1 shows the clusters and items being allocated to the clusters [34], [35].

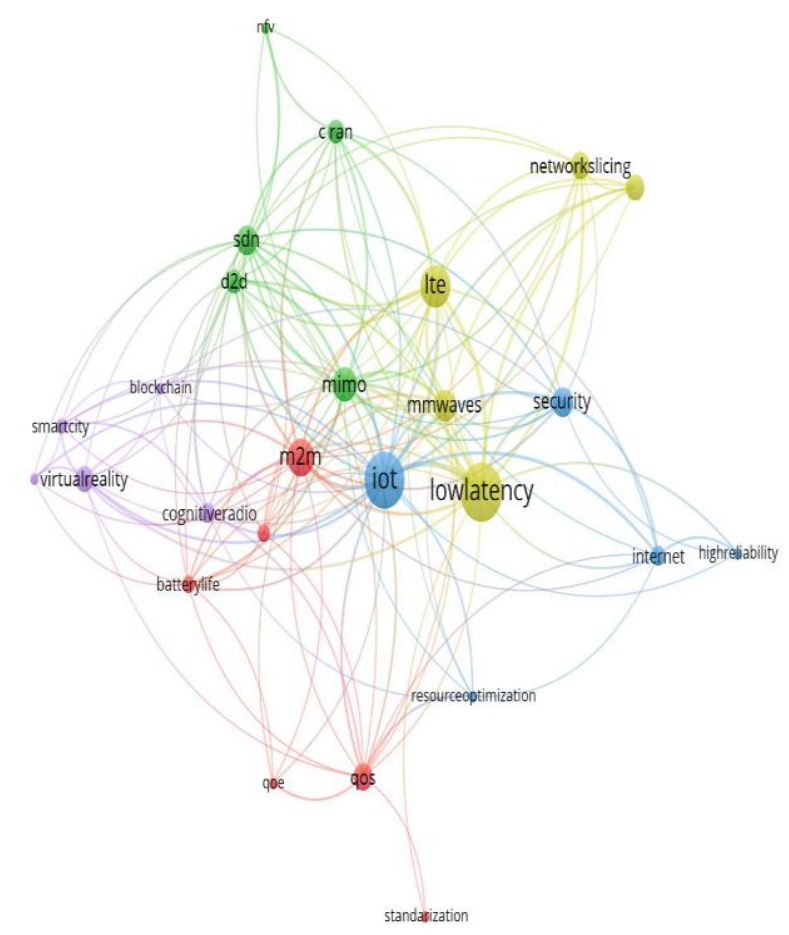

Fig.-3 Co-occurrence network of keywords related to 5G
Table-1 Cluster wise allocation of the items

\begin{tabular}{|c|c|c|}
\hline $\begin{array}{l}\text { Cluster } \\
\text { Name }\end{array}$ & Keywords & $\begin{array}{c}\text { Occurrences } \\
\quad(N=65)\end{array}$ \\
\hline \multirow{6}{*}{$\mathrm{C} 1$} & Battery life & 4 \\
\hline & $\mathrm{M} 2 \mathrm{M}$ & 13 \\
\hline & QoE & 2 \\
\hline & QoS & 8 \\
\hline & Standardization & 2 \\
\hline & $\begin{array}{l}\text { User } \\
\text { Experience }\end{array}$ & 4 \\
\hline \multirow{5}{*}{$\mathrm{C} 2$} & C-RAN & 6 \\
\hline & D2D & 6 \\
\hline & MIMO & 11 \\
\hline & NFV & 2 \\
\hline & SDN & 9 \\
\hline \multirow{5}{*}{$\mathrm{C} 3$} & $\begin{array}{l}\text { High } \\
\text { Reliability }\end{array}$ & 2 \\
\hline & Internet & 5 \\
\hline & IoT & 24 \\
\hline & $\begin{array}{l}\text { Resource } \\
\text { Optimization }\end{array}$ & 2 \\
\hline & Security & 9 \\
\hline \multirow{5}{*}{$\mathrm{C} 4$} & Block chain & 2 \\
\hline & $\begin{array}{l}\text { Cognitive } \\
\text { Radio }\end{array}$ & 5 \\
\hline & $\begin{array}{l}\text { Intelligent } \\
\text { Manufacturing }\end{array}$ & 2 \\
\hline & Smart City & 3 \\
\hline & Virtual Reality & 7 \\
\hline \multirow{5}{*}{ C5 } & Low Latency & 27 \\
\hline & LTE & 16 \\
\hline & mmWaves & 10 \\
\hline & $\begin{array}{l}\text { Network } \\
\text { Slicing }\end{array}$ & 8 \\
\hline & Spectrum & 7 \\
\hline
\end{tabular}

\section{Objective5: To understand Key challenges for implementing 5G.}

Just like any new technology, $5 \mathrm{G}$, too, is going to have its share of challenges. In last 40 years of moving from $1 \mathrm{G}$ to $5 \mathrm{G}$, the fundamental challenges have remained more or less same such as high infrastructure cost, security \& privacy, high switching cost for customers and compatibility of old technology with new one. Moreover, there are countries that are still on $2 \mathrm{G}$ and $3 \mathrm{G}$ mobile networks and are yet to even experience 4G [1], [2]. Also, even those countries where 4G 
is launched, it is yet to reach its full capacity. In such a situation, 5G technology as a global mobile communication remains a more than a challenge. In addition, there are very specific challenges related to $5 \mathrm{G}$ technology given as below -

a) Technological challenges - 5G technology is different from its predecessor $4 \mathrm{G}$ in terms of smaller cell replacing macro-cells. Since there will be difference in the size of these cells so it is going to create an issue of interference. Further, in 5G, there access points will be very dense which may cause low throughput and high latency. Also, 5G is likely to make machine-to machine conversation feasible, the interaction between hundreds and thousands of different types of devices could be extremely damaging and therefore requires proper research and testing [3].

b) Other Challenges - Apart from technological challenges, $5 \mathrm{G}$ is likely to face variety of other issues such as Govt. regulations and cyber laws of a particular country, infrastructure development and maintenance requirements, Security and privacy, standardization of services, spectrum issues and Social impact of $5 \mathrm{G}$ on flora- fauna and society in general

\section{Conclusion, Limitations and future scope}

This study was an attempt to understand what $5 \mathrm{G}$ mobile communication technology is and how it is different and better from its predecessor $4 \mathrm{G}$. This article also discusses the $5 \mathrm{G}$ architecture requirements and covers how potential issues that may come up during $5 \mathrm{G}$ technology implementation. $5 \mathrm{G}$ technology comes as a far better alternative to $4 \mathrm{G}$ which may have reached it capacity to meet fast communication data requirements of today's users. With features such as high data rate, low latency rate and better geographical coverage, $5 \mathrm{G}$ holds a lot promise as a next generation technology. Although, 5G is indeed a technology for future but it is not going to be easy to implement. There are several issues that need to be sorted out before users can adopt it. Issues such as infrastructure development, research based issue handling, security and privacy, spectrum requirements, switching cost of moving from $4 \mathrm{G}$ to $5 \mathrm{G}$, other social implications need to be sorted out before $5 \mathrm{G}$ is comprehensively adopted. The current study is qualitative attempt to understand $5 \mathrm{G}$ technology and its related issues. It suffers from lot of limitations at this stage as it very early and there is not enough research done on several aspects related to its implementation. The study only discusses articles available from 2010 onwards as there were very few articles available prior to this time. Further, this study does not take up completely technical articles as that is beyond the scope of this study. Methodology adopted in this article is critical content analysis which may not be sufficient enough to reach conclusion. In future, the study can be improved by taking all possible articles on $5 \mathrm{G}$ covering technical as well as non-technical articles. Further, a mixed methodology may be adopted to reach out to more concrete conclusions

\section{References}

1. Agarwal, A., Misra, G., Agarwal, S. and Ghosh, K., 2019. 5G Wireless Cellular Networks: A Conceptual Analysis on Perception, Network Requirements and Enabling Technologies. Journal of The Institution of Engineers (India): Series B, 100(2), pp.187-191.

2. Recommendation ITU-R M.2083-0, IMT-Vision, Framework and overall objectives of the future development of IMT for 2020 and Beyond (2015)

3. A Report, Ericsson Mobility Report: 5G uptake even faster than expected, Accessed on $1^{\text {st }}$ Jan 2020

https://www.ericsson.com/en/pressreleases/2019/6/ericsson-mobility-report-5g-uptakeeven-faster-than-expected

4. White paper, 17 predictions about $5 \mathrm{G}$ networks and devices, Accessed on : 1st Jan 2020

https://www.networkworld.com/article/3403358/17predictions-about-5g-networks-and-devices.html

5. FN Division, TEC, (2019),"5G-Key Capabilities \& Applications", white paper

6. Enabling 5G in India- TRAI white paper: https://main.trai.gov.in/notifications/press-release/traireleases-white-paper-enabling-5g-india

7. Davaslioglu, K. and Ayanoglu, E., 2014. Quantifying potential energy efficiency gain in green cellular wireless networks. IEEE Communications Surveys \& Tutorials, 16(4), pp.2065-2091.

8. A report of Department of Telecommunications, Making India 5G Ready- 5H HLF, Accessed on : $1^{\text {st }}$ Jan 2020. http://dot.gov.in/whatsnew/making-india-5g-readyreport-5g-high-level-forum

9. Badoi, C.I., Prasad, N., Croitoru, V. and Prasad, R., 2011. 5G based on cognitive radio. Wireless Personal Communications, 57(3), pp.441-464.

10. Sayrafian, K. and Yazdandoost, K.Y., 2015. Toward 5G Emerging Technologies: Selected Papers from IEEE PIMRC 2014. International Journal of Wireless Information Networks, 22(4), pp.295-297. 
11. Mastrosimone, A. and Panno, D., 2018. Moving network based on mmWave technology: a promising solution for $5 \mathrm{G}$ vehicular users. Wireless Networks, 24(7), pp.24092426.

12. Kaur, K., Kumar, S. and Baliyan, A., 2018. 5G: a new era of wireless communication. International Journal of Information Technology, pp.1-6.

13. Ji, X., Huang, K., Jin, L., Tang, H., Liu, C., Zhong, Z., You, W., Xu, X., Zhao, H., Wu, J. and Yi, M., 2018. Overview of $5 \mathrm{G}$ security technology. Science China Information Sciences, 61(8), p.081301.

14. Chu, Y., Pan, L., Leng, K., Fu, H.C. and Lam, A., Research on key technologies of service quality optimization for industrial IoT $5 \mathrm{G}$ network for intelligent manufacturing. The International Journal of Advanced Manufacturing Technology, pp.1-10.

15. Awoyemi, B.S., Alfa, A.S. and Maharaj, B.T., Resource Optimisation in $5 \mathrm{G}$ and Internet-of-Things Networking. Wireless Personal Communications, pp.132.

16. Rendon Schneir, J., Ajibulu, A., Konstantinou, K., Bradford, J., Zimmermann, G., Droste, H. and Canto, R., 2019. A business case for $5 \mathrm{G}$ mobile broadband in a dense urban area. Telecommunications Policy, 43(7), pp.1-1.

17. Mistry, I., Tanwar, S., Tyagi, S. and Kumar, N., 2020. Blockchain for 5G-enabled IoT for industrial automation: A systematic review, solutions, and challenges. Mechanical Systems and Signal Processing, 135, p.106382.

18. Tranfield, D., Denyer, D., Smart, P. 2003. Towards a methodology for developing evidence-informed management knowledge by means of systematic review. British Journal of Management, 14(3), 207-222.

19. Reay, T., Berta, W. and Kohn, M.K., 2009. What's the evidence on evidence-based management? Academy of Management Perspectives, 23(4), 5-18.

20. Zhu, Q., Sarkis, J., 2016. Green marketing and consumerism as social change in China: Analyzing the literature. International Journal of Production Economics, 181, 289-302.

21. Beske, P., Land, A., Seuring, S. 2014. Sustainable supply chain management practices and dynamic capabilities in the food industry: A critical analysis of the literature. International Journal of Production Economics, 152, 131-143.

22. Sunder M, V., Ganesh, L.S., Marathe, R. R. 2018. A morphological analysis of research literature on Lean Six
Sigma for services. International Journal of Operations \& Production Management, 38(1), 149-182.

23. Macnamara, J.R., 2005. Media content analysis: its uses, benefits and best practice methodology. Asia Pacific Public Relations Journal. 6 (1), 1.

24. Newbold, C., Boyd-Barrett, O., Van Den Bulck, H., 2002. The Media Book. Arnold (Hodder Headline), London.

25. Carvalho, Anabela, 2008. Media(ted) discourse and society. Journal. Stud. 9 (2), 161-177.

26. Johnson, H., Mathis, J. and Short, K.G., 2016. Critical content analysis of children's and young adult literature. New York, NY: Routledge.

27. Definitive data and analysis for the mobile industry, GSMA intelligence 2019, Accessed on: 1st Jan 2020. https://www.gsmaintelligence.com/

28. Zhang, P., 2017. Special issue on 5G wireless communication systems and technologies, pp. 751-752.

29. Li, S., Da Xu, L. and Zhao, S., 2018. 5G Internet of Things: A survey. Journal of Industrial Information Integration, 10, pp.1-9.

30. Farooq, M.U., Waseem, M., Qadri, M.T. and Waqar, M., 2017. Understanding 5G wireless cellular network: Challenges, emerging research directions and enabling technologies. Wireless Personal Communications, 95(2), pp.261-285.

31. Tang, Z., Jayakar, K., Feng, X., Zhang, H. and Peng, R.X., 2019. Identifying smart city archetypes from the bottom up: A content analysis of municipal plans. Telecommunications Policy, 43(10), p.101834.

32. Ciano, M.P., Pozzi, R., Rossi, T. and Strozzi, F., 2019. How IJPR has addressed 'lean': a literature review using bibliometric tools. International Journal of Production Research, pp.1-34.

33. Waltman, L., Van Eck, N.J. and Noyons, E.C., 2010. A unified approach to mapping and clustering of bibliometric networks. Journal of Informetrics, 4(4), pp.629-635.

34. Prashar, A., 2019. A bibliometric and content analysis of sustainable development in small and medium-sized enterprises. Journal of Cleaner Production, p.118665.

35. Pollack, J. and Adler, D., 2015. Emergent trends and passing fads in project management research: A scientometric analysis of changes in the field. International Journal of Project Management, 33(1), pp.236-248. 